\title{
The preoperative incidence of raised intracranial pressure in nonsyndromic sagittal craniosynostosis is underestimated in the literature
}

\author{
Clinical article
}

\author{
*Steven A. Wall, M.B., Ch.B., Gregory P. L. Thomas, B.M., B.CH., Ph.D., \\ David Johnson, B.CH., D.M., Jo C. Byren, M.B., B.S., Jayaratnam Jayamohan, M.B., B.S., \\ Shailendra A. Magdum, M.B., M.S., David J. McAuley, M.B., B.Ch., \\ and Peter G. Richards, M.B., B.S. \\ Oxford Craniofacial Unit, Oxford University Hospitals National Health Service Trust, Oxford, United \\ Kingdom
}

Object. The presence of raised intracranial pressure (ICP) in untreated nonsyndromic, isolated sagittal craniosynostosis (SC) is an important functional indication for surgery.

Methods. A retrospective review was performed of all 284 patients presenting with SC to the Oxford Craniofacial Unit between 1995 and 2010.

Results. Intraparenchymal ICP monitoring was performed in 39 children following a standard unit protocol. Monitoring of ICP was offered for all patients in whom nonoperative management was considered on the basis of minimal deformity or in cases in which parents were reluctant to agree to corrective surgery. These patients presented at an older age than the rest of the cohort (mean age 56 months), with marked scaphocephaly $(16 / 39,41 \%)$, mild scaphocephaly $(11,28 \%)$, or no scaphocephalic deformity $(12,31 \%)$. Raised ICP was found in $17(44 \%)$ patients, with no significant difference in its incidence among the 3 different deformity types. Raised ICP was not predicted by the presence of symptoms of ICP or developmental delay or by ophthalmological or radiological findings.

Conclusions. The incidence of raised ICP in SC reported here is greater than that previously published in the literature. The lack of a reliable noninvasive method to identify individuals with elevated ICP in SC mandates consideration of intraparenchymal ICP monitoring in all patients for whom nonoperative management is contemplated. (http://thejns.org/doi/abs/10.3171/2014.8.PEDS1425)

\section{KEY WORDS • intracranial pressure • intracranial hypertension • sagittal craniosynostosis $\quad$ craniofacial}

$\mathrm{S}$ INGLE-suture nonsyndromic sagittal craniosynostosis (SC) is the most common form of craniosynostosis, affecting between 1 in 2000 and 1 in 5000 children, with a 4:1 male-to-female ratio..$^{30,32,56}$ Most patients present in infancy or early childhood with a scaphocephalic head shape and associated calvarial abnormalities. ${ }^{10}$ Historically this morphological deformity alone has been a sufficient indication for corrective transcranial surgery. Furthermore, it is now recognized that raised intracranial pressure (ICP) occurs in this group of patients, with an estimated preoperative incidence between $4.5 \%$ and $24 \% .6,22,40,41,49,50$ Therefore, the treatment of possible preexisting raised ICP, or the prevention of future ICP, provides a clear functional indication for surgery, in ad-

Abbreviations used in this paper: $\mathrm{ICP}=$ intracranial pressure; $\mathrm{SC}$ $=$ sagittal craniosynostosis.

* Drs. Wall and Thomas contributed equally to this work. dition to the need to correct the morphological deformity present in these children.

However, a number of patients have only mild scaphocephalic deformity or no scaphocephaly at all, despite having SC. The aesthetic indications for corrective surgery in this group are much weaker, or absent, and parents are likely to be understandably more reluctant to agree to a transcranial procedure with its attendant risks for a minor aesthetic benefit. The incidence of raised ICP in these patients has not been previously reported in the literature, although a recent study conducted by our unit in 6 nonscaphocephalic SC patients who underwent ICP monitoring showed that $4(67 \%)$ had elevated ICP. ${ }^{36}$ This high rate may reflect an underlying increased risk of raised ICP in mild or nonscaphocephalic patients compared with the $\mathrm{SC}$ population as a whole. Alternately, it may be a function of the patients' age (mean 46 months), given that Renier et al. ${ }^{41}$ previously found a correlated increase in ICP 
with age, up to 6 years of age, in craniosynostosis. Whatever the etiology, a high rate of raised ICP in SC patients with mild deformity who are less likely to undergo a corrective procedure is potentially significant for those affected individuals. We therefore reviewed a single cohort of patients with nonsyndromic isolated SC to investigate the incidence and associations of elevated ICP in children who did not undergo corrective surgery.

\section{Methods}

A retrospective review was performed of the medical records of all patients presenting with isolated SC to the Oxford Craniofacial Unit between January 1995 and December 2010. All patients included in the study had a diagnosis of isolated SC confirmed on radiological imaging and were managed by the unit. The following were excluded: 1) individuals who transferred their care to another unit after their initial visit or who failed to attend all subsequent investigations and clinic appointments; 2) patients identified on screening to have either genetic mutations or syndromic conditions known to be associated with craniosynostosis; and 3) patients with SC considered secondary to perinatal intraventricular hemorrhage, hydrocephalus, or CSF shunting procedures. The study was conducted in accordance with local ethics committee regulations.

In those patients in whom it was performed, ICP monitoring was conducted over a 24- to 48-hour period using an intraparenchymal Codman MicroSensor (Johnson \& Johnson Professionals, Inc.) placed in the right or left frontal lobe. An ICP with a baseline consistently above $15 \mathrm{~mm} \mathrm{Hg}$ or more than 3 B-type waves in a 24hour period during sleep was classified as raised. ${ }^{55}$

Statistical analysis was performed using the Minitab statistical software package (version 16; Minitab Inc.). The outcome of interest was whether a patient had raised ICP. Predictors of interest included age at surgery (months), scaphocephalic deformity (marked, mild, or none), symptoms of raised ICP, developmental delay, ophthalmological findings, and radiographic findings. Univariate logistic regression modeling was used to describe the association of each predictor with the outcome.

\section{Results}

Between 1995 and 2010, 284 new SC patients (217 male, 67 female) were seen. Generally, infants presenting before 6 months of age underwent a modified sagittal strip procedure, whereas those presenting after 6 months of age underwent calvarial remodeling. Ninety-four patients (33\%) underwent modified strip craniectomy (mean age at presentation 4.2 months), and 139 patients (49\%) underwent calvarial remodeling (mean age at presentation 10.8 months).

The remaining 51 patients $(18 \%)$ comprised 6 adults and 45 children. The adults presented at a mean age of 24.4 years (range 18.6-37.2 years), all having failed to have been diagnosed with scaphocephaly in childhood. One adult had experienced a long history of recurrent headaches with episodes of vomiting and was found to have marked beaten-copper appearance of the calvaria on radiological imaging. He underwent ICP monitoring, and the results were normal. All 6 adults were discharged without further surgical intervention.

The 45 children presented at a later mean age (56 months, range 9-163 months) than the rest of the cohort (Fig. 1). Twenty-six patients had either nonscaphocephalic SC (cephalic index $>75 ; n=13$ ) or clinically mild scaphocephaly (cephalic index 70-75; $n=13$ ); in none of these cases was surgical correction thought to be warranted on aesthetic grounds alone. Nineteen patients had a significant scaphocephalic deformity (cephalic index $<70$ ), but either the parents were unwilling to agree to transcranial surgery for aesthetic reasons only $(n=15)$ or there was a relative contraindication to surgery either from a preexisting comorbidity $(n=1)$ or from the religious beliefs of the family preventing the use of blood transfusion should the need arise $(n=3)$.

Nonscaphocephalic patients had SC diagnosed based on radiological imaging performed for an abnormal head shape, including posterior plagiocephaly, brachycephaly, turricephaly with frontal bossing, a metopic ridge, a saddle deformity of the vertex with an occipital bullet, microcephaly, and macrocephaly. One patient had SC diagnosed coincidentally when imaged for an unrelated scalp

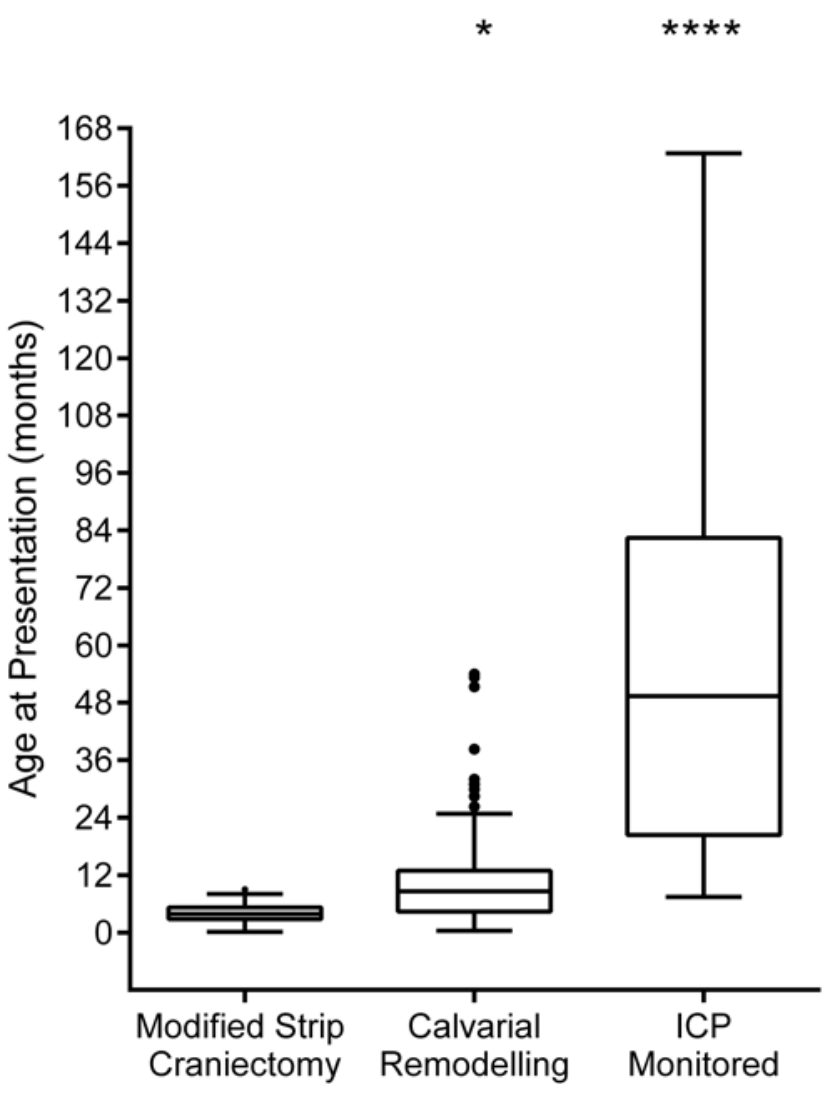

FIG. 1. Tukey box-and-whisker plot of age at presentation in children with SC. The ICP-monitored group also includes 6 patients who were offered ICP monitoring but declined. Mean age of the ICP-monitored group was significantly greater than means of the other 2 groups $\left({ }^{* * *} p\right.$ $<0.0001$ ), and mean age of the calvarial remodeling group was greater than that of the strip craniectomy group ( ${ }^{*} p<0.05$, Tukey's multiple comparison test). 
lesion. Another child was imaged because his mother was known to carry a fibroblast growth factor receptor mutation, although this was excluded in the patient by genetic analysis.

Five patients, 3 with a mild scaphocephaly and 2 with a significant scaphocephaly, presented with sufficiently marked developmental delay for parents and the craniofacial team to question whether the child would gain any psychosocial benefit from a more normal head shape after surgery. Moderate developmental delay was identified in an additional 9 patients. All patients presenting with a developmental delay were encouraged to have ICP monitoring because of the concern that their developmental delay might be associated with raised ICP.

Eight patients manifested possible symptoms of raised ICP at presentation: persistent headaches ( $\mathrm{n}=$ 1), nocturnal wakening in distress with head holding or banging (sleep disturbance; $n=2$ ), or deteriorating attention span with a decline in academic attainment (irritability; $n=5$ ). Two of these children also had a developmental delay. All patients underwent an ophthalmological assessment. None were found to have papilledema.

Cranial CT scans demonstrated signs suggestive of raised ICP, including diffuse beaten-copper appearance of the calvaria, a reduction in the volume of the extraaxial subarachnoid spaces, or effacement of the sulci, in 30 patients who went on to have ICP monitoring. Fourteen patients with positive radiological findings proved to have raised ICP on monitoring; the remaining 16 patients had a normal ICP. Overall, radiological investigations had a sensitivity of $82 \%$ and a specificity of $27 \%$. When all signs other than beaten-copper appearance were considered together, sensitivity declined to $65 \%$ but specificity rose to $64 \%$. Monitoring of ICP was considered for all patients as a routine part of the preoperative decisionmaking protocol. Upon review, 4 patients with no history of developmental delay or symptoms of raised ICP and normal radiological findings were deemed not to require ICP monitoring. An additional patient who had only mild beaten-copper appearance of the calvaria on imaging but otherwise was developing well and had no signs or symptoms suggestive of raised ICP was viewed not to need monitoring. One child was offered ICP monitoring, which was declined by the family. Of these 6 patients, 4 continue to undergo follow-up and 2 were discharged at 16 years of age. All remained well and asymptomatic when last reviewed.

A total of 39 children underwent ICP monitoring at a mean age of 65 months (range 17-169 months) (Table 1). Of these, 17 (44\%; 95\% CI 28\%-59\%) were found to have elevated ICP (Tables 1 and 2); one had a normal initial ICP but after a progressive deterioration in behavior was found to have elevated ICP on monitoring 30 months later (Table 2, Case 13). Another patient had borderline raised ICP with an overnight baseline of $15 \mathrm{~mm} \mathrm{Hg}$ and 2 B-waves (Table 2, Case 6). The incidence of raised ICP was higher in patients with mild or absent deformity (64\% and 43\%, respectively) than in those with marked scaphocephaly (31\%), although this was not statistically significant (Tables 1 and 3). The incidence of raised ICP was not affected by the age of the patient $(\mathrm{F}=0.24, \mathrm{p}=$
0.63 , linear regression analysis). The presence of neither preoperative symptoms nor radiological signs suggested raised ICP, and developmental delay was not significantly more common in patients with elevated ICP (Table 3 ).

All patients with abnormal ICP underwent calvarial remodeling, including the child with nonscaphocephalic $\mathrm{SC}$ and borderline ICP. Five children with ICP in the normal range underwent calvarial remodeling. One child with mild scaphocephaly became clinically more scaphocephalic over time, and surgery was performed at the parents' request. Four children with marked scaphocephaly also underwent corrective surgery at their parents' request.

Among patients who underwent calvarial remodeling for raised ICP, 6 patients underwent a further episode of ICP monitoring for new symptoms or signs suggestive of raised ICP at a mean of 49 months after their primary remodeling procedure (Table 2). None proved to have raised ICP. One further patient who had normal initial ICP but underwent calvarial remodeling for marked scaphocephaly later developed morning headaches, irritability, and a decline in academic performance. She had repeat ICP monitoring at 39 months after her primary surgery, and her ICP was found to be normal.

All 39 children who underwent ICP monitoring continue to undergo follow-up. Patients who manifested symptoms suggestive of raised ICP but who were found to have normal ICP on monitoring experienced an improvement or resolution of their symptoms over time. Computed tomography was not routinely performed during follow-up to minimize radiation exposure. No patient has required a repeat calvarial remodeling procedure.

\section{Discussion}

The overall incidence of raised ICP in patients who underwent ICP monitoring in this study was $44 \%$. If the 6 patients in whom ICP monitoring was considered but not performed are taken into account, assuming a negative ICP reading in those cases, the rate falls to $38 \%$. This incidence is still greater than that observed in other series of nonoperated scaphocephalic patients. 4,6,22,40,41,49,50 This difference may be explained by either the greater age of our patients or the high proportion of mild and nonscaphocephalic patients in the group.

Of the published series, only Arnaud et al. ${ }^{6}$ reported the mean age of their scaphocephalic patients at the time of ICP monitoring; their mean age was 1 year and the incidence of elevated ICP was $13.4 \%$. The mean age of patients in our study was 4 years 8 months. Renier et al. ${ }^{41}$ noted a rise in ICP with age to 6 years, and Arnaud et al. ${ }^{6}$ found a significantly increased incidence of raised ICP in patients older than 1 year compared with younger patients. Seruya et al..$^{43}$ reported intracranial hypertension in $9(82 \%)$ of 11 patients who underwent calvarial remodeling at a mean age of 40.5 months for delayed-presentation SC. Conversely, Thompson et al. ${ }^{50}$ found an inverse relationship between age and ICP in scaphocephaly. In our patient group, age was not associated with raised ICP. However, given the older age of the group as a whole and the $31 \%$ incidence of raised ICP among patients with 
Raised ICP in sagittal synostosis prior to surgery

TABLE 1: Children who underwent ICP monitoring

\begin{tabular}{|c|c|c|c|c|c|c|c|c|}
\hline \multirow[b]{2}{*}{ Deformity* } & \multirow[b]{2}{*}{$\begin{array}{l}\text { No. of } \\
\text { Patients }\end{array}$} & \multirow{2}{*}{$\begin{array}{l}\text { Mean Age at } \\
\text { Presentation } \\
\text { (mos) }\end{array}$} & \multirow{2}{*}{$\begin{array}{l}\text { Mean } \\
\text { Cephalic } \\
\text { Index }\end{array}$} & \multicolumn{5}{|c|}{ Characteristic $\dagger$} \\
\hline & & & & $\begin{array}{l}\text { Developmental } \\
\text { Delay }\end{array}$ & $\begin{array}{l}\text { Symptoms of } \\
\text { Raised ICP }\end{array}$ & $\begin{array}{l}\text { Radiological Signs } \\
\text { of Raised ICP }\end{array}$ & $\begin{array}{l}\text { Raised ICP } \\
(\%)\end{array}$ & $\begin{array}{c}\text { Calvarial } \\
\text { Remodeling }\end{array}$ \\
\hline marked scaphocephaly & 16 & 58 & 66.2 & 8 & 1 & 13 & $5(31)$ & 9 \\
\hline mild scaphocephaly & 11 & 43 & 72.4 & $3 \ddagger$ & 3 & 9 & $7(64)$ & 8 \\
\hline nonscaphocephalic & 12 & 65 & 84.4 & $3 \ddagger$ & 4 & 8 & $5(42) \S$ & 6 \\
\hline total & 39 & 56 & 73.5 & $14 \ddagger$ & 8 & 30 & $17(44)$ & 23 \\
\hline
\end{tabular}

* Marked scaphocephaly = cephalic index $<70$; mild scaphocephaly $=$ cephalic index 70 -75 ; nonscaphocephalic $=$ cephalic index $>75$.

$\dagger$ Values are the number of patients with the characteristic.

$\ddagger$ Two patients, 1 with a mild scaphocephaly and 1 nonscaphocephalic, had developmental delay related to cerebral palsy.

$\S$ An additional patient in this group had borderline elevated ICP and subsequently underwent calvarial remodeling.

marked scaphocephaly, which is higher than other published rates, the incidence of abnormal ICP may well increase with age in this population. This view is supported by the 1 patient in this study who, after a normal ICP recording, suffered behavioral deterioration and was found to have raised ICP more than 2 years later.

Although some questions remain as to what constitutes normal ICP in infants, it is broadly accepted that adult thresholds are appropriate in children older than 1 year, who have more rigid calvaria, when assessing ICP in craniosynostosis. ${ }^{6,20,35,41,49,50,54,55}$ There were no infants in this study; the youngest child was 17 months of age at ICP monitoring.

Previous studies that have performed overnight ICP monitoring in craniosynostosis have all defined abnormally raised ICP, or intracranial hypertension, as a baseline ICP of $15 \mathrm{~mm} \mathrm{Hg}$ or greater, irrespective of whether an extradural or subdural device was used. $6,20,22,36,41,49-52$ The pathological significance of multiple B-type waves is also well recognized. ${ }^{20,41,55}$ An upper normal threshold of $15 \mathrm{~mm} \mathrm{Hg}$ is commonly used in other chronic conditions, including hydrocephalus, ${ }^{12,55}$ although some authors rely on a higher threshold of $20 \mathrm{~mm} \mathrm{Hg}$, such as in the case of idiopathic ICP. ${ }^{24}$ If the higher threshold of $20 \mathrm{~mm} \mathrm{Hg}$ were used to define raised ICP in this study, 6 of 39 patients $(15 \%)$ would have been deemed to have raised ICP.

Intraparenchymal ICP monitoring remains the most reliable and reproducible method at our disposal to assess ICP and is associated with an infrequent and minor complication rate. ${ }^{20,31,39,55}$ The lower rate of raised ICP observed by Renier et al. ${ }^{41}$ may be due to their use of a less sensitive extradural method of monitoring. ${ }^{11}$

Milder deformities in single-suture craniosynostosis have been associated with high rates of elevated ICP. ${ }^{21,27}$ Consistent with those reports, the highest rate in this study was observed in mildly scaphocephalic patients (64\%). Whether age at presentation or the type of deformity is more significant in the development of raised ICP is debatable, because children with milder deformities are more likely to present at an older age.

Twelve of 45 children $(27 \%)$ in our study presented with some degree of neurodevelopmental delay or learning difficulties not attributable to other factors. This rate is consistent with findings of several studies that examined developmental attainment, learning disability, and speech and language abnormalities in isolated SC. $7,8,13,14,33,44,46 \mathrm{Al}-$ though, in 1982, Renier et al ${ }^{41}$ found that raised ICP was correlated with lower IQ, more recent studies ${ }^{6,23,34}$ have failed to confirm the relationship between elevated ICP and neurocognitive outcomes in SC or other single-suture craniosynostosis. The lack of a correlation between developmental delay and raised ICP found in the present study supports the hypothesis that there is no simple causal relationship between ICP and neurocognitive anomalies found in SC. ${ }^{25}$ Three-dimensional imaging studies performed by Aldridge et al. ${ }^{2,3}$ indicate that primary structural CNS abnormalities are present in some patients with $\mathrm{SC}$, suggesting an alternative explanation for some of the neurocognitive abnormalities observed. ${ }^{29}$

Whatever the ultimate cause of neurocognitive abnormalities proves to be in SC, elevated ICP should not be dismissed as a benign finding without possible functional consequences for the individual. The sequelae of chronically raised ICP include visual loss and psychomotor impairment. ${ }^{38,53}$ In other pediatric neurological conditions, elevated ICP has been correlated with impaired myelination and later poor neurodevelopmental scores. ${ }^{26}$ Further, several PET studies in children with isolated single-suture craniosynostosis have found areas of cerebral hypoperfusion associated with the stenosed suture, which resolve after corrective surgery. ${ }^{16,17,42}$

In the literature, only Arnaud et al. ${ }^{6}$ measured ICP in a population that had not undergone corrective surgery and then followed their neurocognitive development over time. They found a significant correlation between developmental quotient and final IQ of patients who had avoided surgery. All patients with proven raised ICP (n $=4$ ) had an IQ exceeding 90 on final assessment. Interestingly, however, the correlation between developmental quotient and final IQ was much poorer in patients who did not undergo surgery $(r=0.3)$ than in those who did undergo surgery $(r=0.64)$. Perhaps the reduced correlation observed in the nonsurgical group could be accounted for by either the patients with raised ICP declining in IQ, though not to the threshold of 90 , or some patients with initially normal ICP subsequently developing raised ICP and experiencing a relative drop in IQ.

Several studies have found no significant improvement in neurodevelopmental outcomes after corrective surgery. ${ }^{13,28,45,47}$ However, it is difficult to infer anything 
S. A. Wall et al.

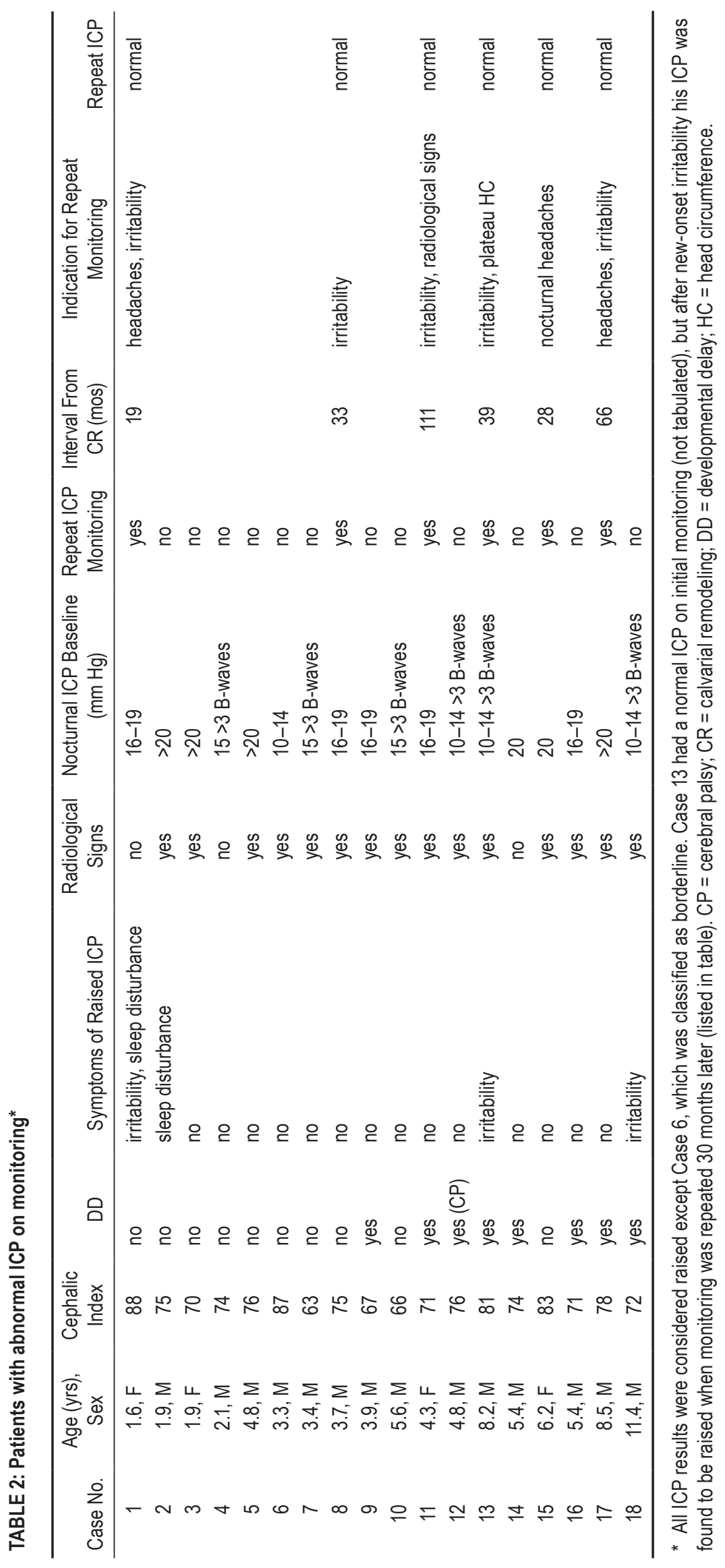


TABLE 3: Symptoms and clinical signs associated with raised ICP in 39 patients

\begin{tabular}{|c|c|c|c|c|}
\hline \multirow[b]{2}{*}{ Symptoms/Signs } & \multicolumn{2}{|c|}{ No. of Patients } & \multirow[b]{2}{*}{ OR } & \multirow[b]{2}{*}{$\mathrm{p}$ Value } \\
\hline & $\begin{array}{l}\text { Raised ICP } \\
\qquad(n=17)\end{array}$ & $\begin{array}{l}\text { Normal ICP } \\
\quad(n=22)\end{array}$ & & \\
\hline \multicolumn{5}{|l|}{ scaphocephaly } \\
\hline marked & 5 & 11 & & \\
\hline mild & 7 & 4 & 3.85 & \\
\hline absent & 5 & 7 & 1.57 & 0.24 \\
\hline \multicolumn{5}{|l|}{ developmental delay* } \\
\hline present & 7 & 5 & & \\
\hline absent & 10 & 16 & 2.38 & 0.22 \\
\hline \multicolumn{5}{|c|}{ symptoms of raised ICP } \\
\hline present & 4 & 4 & & \\
\hline absent & 14 & 18 & 1.38 & 0.68 \\
\hline \multicolumn{5}{|l|}{$\begin{array}{l}\text { radiological signs of } \\
\text { raised ICP }\end{array}$} \\
\hline present & 14 & 16 & & \\
\hline absent & 3 & 6 & 2.18 & 0.31 \\
\hline
\end{tabular}

* Two patients with cerebral palsy were excluded from this group. $\dagger$ Logistic regression analysis.

directly from these observations without corresponding ICP data, because in the authors' and others' experience, raised ICP can occur after surgical corrective procedures. ${ }^{9}$ Any resultant decline in neurocognitive function in affected individuals could mask an improvement in the overall group. Furthermore, there is contrary evidence that, after surgery, single-suture craniosynostosis patients are of normal intelligence during school-age years and patients with a proven raised preoperative ICP experience improved neurocognitive functioning. ${ }^{15,27}$ With the evidence currently available in the literature, it remains reasonable to assume that continued chronically raised ICP in SC is liable to curtail an individual's neurocognitive development, even if it does not reliably cause a marked developmental delay and is not responsible for many of the developmental abnormalities seen in SC.

Symptoms suggestive of ICP, the presence of papilledema, and radiological studies did not usefully predict elevated ICP in this study. Eide et al., ${ }^{19,20}$ in 2 large series of pediatric patients with craniosynostosis, hydrocephalus, shunt failure, or idiopathic ICP, found that symptoms classically associated with raised ICP (headache, irritability, sleep disturbance, nausea, psychomotor delay, and seizures) did not correlate with or predict raised ICP in children, and our findings reflect this. Irritability and sleep disturbance, the most frequently encountered symptoms in our study, have a wide range of possible causes. Papilledema is unusual in children younger than 8 years with raised ICP in the context of craniosynostosis and thus is a sign of low sensitivity but very high specificity. ${ }^{19,51}$ Tuite et al. ${ }^{51}$ hypothesized that this may be due to either the difficulties inherent in examining the optic discs of poorly compliant infants or anatomical or physiological differences between young children and older children and adults. Conversely, radiological signs suggestive of raised ICP, particularly a diffuse beaten-copper appearance of the cranium, are common in children with craniosynostosis and increase in incidence with age. ${ }^{1,52}$ They are thus poorly predictive of raised ICP. ${ }^{52}$

Visual evoked potential monitoring holds promise as a noninvasive technique for assessing ICP in craniosynostosis, but a high incidence of abnormal responses in craniosynostosis patients and variability in normal subjects suggest that currently this approach requires significant neurophysiological expertise. ${ }^{5,18,37,48,55}$ Thus, given the high incidence of raised ICP in this study (44\% overall), we believe that intraparenchymal ICP monitoring remains the only reliable method to identify elevated ICP in SC patients. It should be considered and used routinely in all patients for whom a nonoperative course of management is proposed, no matter the nature or severity of the calvarial deformity.

\section{Conclusions}

Raised ICP is common in late-presenting patients with SC, irrespective of their calvarial deformity. Previous studies that report the rate of raised ICP in SC likely underestimate its incidence in children who do not undergo corrective surgery. Because clinical and radiological findings are unreliable indicators of raised ICP in $\mathrm{SC}$, intraparenchymal monitoring remains the principal method to accurately determine whether patients can appropriately be managed conservatively.

\section{Acknowledgments}

We are grateful to Dr. Andrew Judge, statistician at the Oxford National Institute for Health Research Musculoskeletal Biomedical Research Unit, for his invaluable assistance in the preparation of the manuscript. We thank all the members of the Oxford Craniofacial Unit team for their assistance throughout this study.

\section{Disclosure}

This work was supported by the Oxford Craniofacial Research Fund. The authors report no conflict of interest concerning the materials or methods used in this study or the findings specified in this paper.

Author contributions to the study and manuscript preparation include the following. Conception and design: Thomas, Wall, Johnson, McAuley. Acquisition of data: Thomas, Johnson, Byren, Jayamohan, Magdum, McAuley, Richards. Analysis and interpretation of data: Thomas, Wall, Johnson, Jayamohan, Magdum. Drafting the article: Thomas, Wall. Critically revising the article: all authors. Reviewed submitted version of manuscript: Wall, McAuley. Approved the final version of the manuscript on behalf of all authors: Thomas. Statistical analysis: Thomas. Administrative/ technical/material support: Thomas, Byren. Study supervision: Wall, Richards.

\section{References}

1. Agrawal D, Steinbok P, Cochrane DD: Significance of beaten copper appearance on skull radiographs in children with isolated sagittal synostosis. Childs Nerv Syst 23:1467-1470, 2007

2. Aldridge K, Kane AA, Marsh JL, Yan P, Govier D, Richts- 
meier JT: Relationship of brain and skull in pre- and postoperative sagittal synostosis. J Anat 206:373-385, 2005

3. Aldridge K, Marsh JL, Govier D, Richtsmeier JT: Central nervous system phenotypes in craniosynostosis. J Anat 201:3139,2002

4. Anderson FM, Geiger L: Craniosynostosis: a survey of 204 cases. J Neurosurg 22:229-240, 1965

5. Andersson L, Sjölund J, Nilsson J: Flash visual evoked potentials are unreliable as markers of ICP due to high variability in normal subjects. Acta Neurochir (Wien) 154:121-127, 2012

6. Arnaud E, Renier D, Marchac D: Prognosis for mental function in scaphocephaly. J Neurosurg 83:476-479, 1995

7. Becker DB, Petersen JD, Kane AA, Cradock MM, Pilgram TK, Marsh JL: Speech, cognitive, and behavioral outcomes in nonsyndromic craniosynostosis. Plast Reconstr Surg 116:400407, 2005

8. Bellew M, Chumas P, Mueller R, Liddington M, Russell J: Pre- and postoperative developmental attainment in sagittal synostosis. Arch Dis Child 90:346-350, 2005

9. Cetas JS, Nasseri M, Saedi T, Kuang AA, Selden NR: Delayed intracranial hypertension after cranial vault remodeling for nonsyndromic single-suture synostosis. Clinical article. J Neurosurg Pediatr 11:661-666, 2013

10. Chatterjee JS, Mahmoud M, Karthikeyan S, Duncan C, Dover MS, Nishikawa H: Referral pattern and surgical outcome of sagittal synostosis. J Plast Reconstr Aesthet Surg 62:211215, 2009

11. Cohen SR, Persing JA: Intracranial pressure in single-suture craniosynostosis. Cleft Palate Craniofac J 35:194-196, 1998

12. Czosnyka M, Pickard JD: Monitoring and interpretation of intracranial pressure. J Neurol Neurosurg Psychiatry 75:813821,2004

13. Da Costa AC, Anderson VA, Holmes AD, Lo P, Wray AC, Chong DK, et al: Longitudinal study of the neurodevelopmental characteristics of treated and untreated nonsyndromic craniosynostosis in infancy. Childs Nerv Syst 29:985-995, 2013

14. Da Costa AC, Anderson VA, Savarirayan R, Wrennall JA, Chong DK, Holmes AD, et al: Neurodevelopmental functioning of infants with untreated single-suture craniosynostosis during early infancy. Childs Nerv Syst 28:869-877, 2012

15. Da Costa AC, Walters I, Savarirayan R, Anderson VA, Wrennall JA, Meara JG: Intellectual outcomes in children and adolescents with syndromic and nonsyndromic craniosynostosis. Plast Reconstr Surg 118:175-183, 2006

16. David LR, Genecov DG, Camastra AA, Wilson JA, Argenta LC: Positron emission tomography studies confirm the need for early surgical intervention in patients with single-suture craniosynostosis. J Craniofac Surg 10:38-42, 1999

17. David LR, Wilson JA, Watson NE, Argenta LC: Cerebral perfusion defects secondary to simple craniosynostosis. J Craniofac Surg 7:177-185, 1996

18. Desch LW: Longitudinal stability of visual evoked potentials in children and adolescents with hydrocephalus. Dev Med Child Neurol 43:113-117, 2001

19. Eide PK, Egge A, Due-Tønnessen BJ, Helseth E: Is intracranial pressure waveform analysis useful in the management of pediatric neurosurgical patients? Pediatr Neurosurg 43:472-481, 2007

20. Eide PK, Helseth E, Due-Tønnessen B, Lundar T: Assessment of continuous intracranial pressure recordings in childhood craniosynostosis. Pediatr Neurosurg 37:310-320, 2002

21. Eley KA, Johnson D, Wilkie AO, Jayamohan J, Richards P, Wall SA: Raised intracranial pressure is frequent in untreated nonsyndromic unicoronal synostosis and does not correlate with severity of phenotypic features. Plast Reconstr Surg 130:690e-697e, 2012

22. Gault DT, Renier D, Marchac D, Jones BM: Intracranial pressure and intracranial volume in children with craniosynostosis. Plast Reconstr Surg 90:377-381, 1992
23. Gewalli F, Guimarães-Ferreira JP, Sahlin P, Emanuelsson I, Horneman G, Stephensen H, et al: Mental development after modified pi procedure: dynamic cranioplasty for sagittal synostosis. Ann Plast Surg 46:415-420, 2001

24. Gjerris F, Soelberg Sørensen P, Vorstrup S, Paulson OB: Intracranial pressure, conductance to cerebrospinal fluid outflow, and cerebral blood flow in patients with benign intracranial hypertension (pseudotumor cerebri). Ann Neurol 17:158162,1985

25. Hankinson TC, Fontana EJ, Anderson RC, Feldstein NA: Surgical treatment of single-suture craniosynostosis: an argument for quantitative methods to evaluate cosmetic outcomes. A review. J Neurosurg Pediatr 6:193-197, 2010

26. Hanlo PW, Gooskens RJ, van Schooneveld M, Tulleken CA, van der Knaap MS, Faber JA, et al: The effect of intracranial pressure on myelination and the relationship with neurodevelopment in infantile hydrocephalus. Dev Med Child Neurol 39:286-291, 1997

27. Inagaki T, Kyutoku S, Seno T, Kawaguchi T, Yamahara T, Oshige $\mathrm{H}$, et al: The intracranial pressure of the patients with mild form of craniosynostosis. Childs Nerv Syst 23:14551459,2007

28. Kapp-Simon KA: Mental development and learning disorders in children with single suture craniosynostosis. Cleft Palate Craniofac J 35:197-203, 1998

29. Kapp-Simon KA, Speltz ML, Cunningham ML, Patel PK, Tomita T: Neurodevelopment of children with single suture craniosynostosis: a review. Childs Nerv Syst 23:269-281, 2007

30. Kolar JC: An epidemiological study of nonsyndromal craniosynostoses. J Craniofac Surg 22:47-49, 2011

31. Koskinen LO, Olivecrona M: Clinical experience with the intraparenchymal intracranial pressure monitoring Codman MicroSensor system. Neurosurgery 56:693-698, 2005

32. Lajeunie E, Le Merrer M, Bonaïti-Pellie C, Marchac D, Renier D: Genetic study of scaphocephaly. Am J Med Genet 62:282-285, 1996

33. Magge SN, Westerveld M, Pruzinsky T, Persing JA: Longterm neuropsychological effects of sagittal craniosynostosis on child development. J Craniofac Surg 13:99-104, 2002

34. Mathijssen I, Arnaud E, Lajeunie E, Marchac D, Renier D: Postoperative cognitive outcome for synostotic frontal plagiocephaly. J Neurosurg 105 (1 Suppl):16-20, 2006

35. Minns RA: Intracranial pressure monitoring. Arch Dis Child 59:486-488, 1984

36. Morritt DG, Yeh FJ, Wall SA, Richards PG, Jayamohan J, Johnson D: Management of isolated sagittal synostosis in the absence of scaphocephaly: a series of eight cases. Plast Reconstr Surg 126:572-580, 2010

37. Mursch K, Brockmann K, Lang JK, Markakis E, BehnkeMursch J: Visually evoked potentials in 52 children requiring operative repair of craniosynostosis. Pediatr Neurosurg 29:320-323, 1998

38. Pollack IF, Losken HW, Biglan AW: Incidence of increased intracranial pressure after early surgical treatment of syndromic craniosynostosis. Pediatr Neurosurg 24:202-209, 1996

39. Pople IK, Muhlbauer MS, Sanford RA, Kirk E: Results and complications of intracranial pressure monitoring in 303 children. Pediatr Neurosurg 23:64-67, 1995

40. Renier D, Lajeunie E, Arnaud E, Marchac D: Management of craniosynostoses. Childs Nerv Syst 16:645-658, 2000

41. Renier D, Sainte-Rose C, Marchac D, Hirsch JF: Intracranial pressure in craniostenosis. J Neurosurg 57:370-377, 1982

42. Sen A, Dougal P, Padhy AK, Bhattacharya A, Kumar R, Bal C, et al: Technetium-99m-HMPAO SPECT cerebral blood flow study in children with craniosynostosis. J Nucl Med 36: 394-398, 1995

43. Seruya M, Oh AK, Boyajian MJ, Posnick JC, Keating RF: Treatment for delayed presentation of sagittal synostosis: challenges pertaining to occult intracranial hypertension. Clinical article. J Neurosurg Pediatr 8:40-48, 2011 


\section{Raised ICP in sagittal synostosis prior to surgery}

44. Shipster C, Hearst D, Somerville A, Stackhouse J, Hayward R, Wade A: Speech, language, and cognitive development in children with isolated sagittal synostosis. Dev Med Child Neurol 45:34-43, 2003

45. Speltz ML, Endriga MC, Mouradian WE: Presurgical and postsurgical mental and psychomotor development of infants with sagittal synostosis. Cleft Palate Craniofac J 34:374379, 1997

46. Speltz ML, Kapp-Simon K, Collett B, Keich Y, Gaither R, Cradock MM, et al: Neurodevelopment of infants with singlesuture craniosynostosis: presurgery comparisons with casematched controls. Plast Reconstr Surg 119:1874-1881, 2007

47. Starr JR, Kapp-Simon KA, Cloonan YK, Collett BR, Cradock $\mathrm{MM}$, Buono L, et al: Presurgical and postsurgical assessment of the neurodevelopment of infants with single-suture craniosynostosis: comparison with controls. J Neurosurg 107 (2 Suppl):103-110, 2007

48. Thompson DA, Liasis A, Hardy S, Hagan R, Hayward RD, Evans RD, et al: Prevalence of abnormal pattern reversal visual evoked potentials in craniosynostosis. Plast Reconstr Surg 118:184-192, 2006

49. Thompson DN, Harkness W, Jones B, Gonsalez S, Andar U, Hayward R: Subdural intracranial pressure monitoring in craniosynostosis: its role in surgical management. Childs Nerv Syst 11:269-275, 1995

50. Thompson DN, Malcolm GP, Jones BM, Harkness WJ, Hayward RD: Intracranial pressure in single-suture craniosynostosis. Pediatr Neurosurg 22:235-240, 1995

51. Tuite GF, Chong WK, Evanson J, Narita A, Taylor D, Harkness
WF, et al: The effectiveness of papilledema as an indicator of raised intracranial pressure in children with craniosynostosis. Neurosurgery 38:272-278, 1996

52. Tuite GF, Evanson J, Chong WK, Thompson DN, Harkness WF, Jones BM, et al: The beaten copper cranium: a correlation between intracranial pressure, cranial radiographs, and computed tomographic scans in children with craniosynostosis. Neurosurgery 39:691-699, 1996

53. Vinchon M, Pellerin P, Baroncini M, Wolber A, Dhellemmes P: Non-syndromic oxycephaly and brachycephaly: a review. Childs Nerv Syst 28:1439-1446, 2012

54. Whittle IR, Johnston IH, Besser M: Intracranial pressure changes in craniostenosis. Surg Neurol 21:367-372, 1984

55. Wiegand C, Richards P: Measurement of intracranial pressure in children: a critical review of current methods. Dev Med Child Neurol 49:935-941, 2007

56. Wilkie AO, Wall SA: Craniosynostosis: novel insights into pathogenesis and treatment. Curr Opin Neurol 9:146-152, 1996

Manuscript submitted January 18, 2014.

Accepted August 14, 2014.

Please include this information when citing this paper: published online September 26, 2014; DOI: 10.3171/2014.8.PEDS1425.

Address correspondence to: Gregory Thomas, Ph.D., Oxford Craniofacial Unit, Oxford University Hospitals NHS Trust, Headley Way, Oxford OX3 9DU, United Kingdom. email: gplthomas@ gmail.com. 